\title{
De un paisaje de degradación a un paisaje de conservación: el tránsito hacia un imaginario del bosque austral como entramado patrimonial (Provincia de Valdivia, Chile)
}

\author{
Francisca Vergara-Pinto \\ Universidad de Los Lagos \\ franverggara@gmail.com \\ Noelia Carrasco Henríquez \\ Universidad de Concepción \\ noeliacarrasco@udec.cl
}

Recepción: 03-06-2019

Aceptación: 20-12-2019

Publicación: 22-05-2020

\section{Resumen}

Este artículo explora los imaginarios de la sustentabilidad del bosque nativo a través de un acercamiento etnográfico a los discursos de habitantes del valle del río San Pedro, Provincia de Valdivia (Chile). Desde una perspectiva científico-social, se aborda la complejidad que representa la conservación de ecosistemas fragmentados inmersos en matrices culturales y productivas diversas y desiguales, y que han sido objeto de la implementación de una estrategia de conservación público-privada denominada Paisaje de Conservación Valle Río San Pedro. Se indagó en procesos socioculturales y en los significados y usos atribuidos localmente al bosque y a su sustentabilidad. A partir de los resultados obtenidos, se invita a reflexionar acerca de la noción de entramado patrimonial como concepto que, en contextos similares al de este estudio, puede dar cuenta de una serie de aspectos físicos y simbólicos, materiales e inmateriales, que conviven y cuya interrelación tiene sentido y valor para las personas. De este modo, se propone una mirada para abordar íntegramente la gestión de la conservación del bosque austral atendiendo la historia local y los diversos procesos biológicos, ambientales, económicos y sociales que han dado forma a los territorios.

Palabras clave: paisaje de conservación; sustentabilidad; bosque nativo; matrices culturales; matrices productivas; río San Pedro; entramado patrimonial 
Abstract. From a landscape of degradation to a landscape of conservation: The transition to an imaginary of the austral forest as a heritage weft (Province of Valdivia, Chile)

This article explores the imaginary of the sustainability of the native forest, through an ethnographic approach to the discourses of inhabitants of the valley of the San Pedro River, Province of Valdivia (Chile). It addresses the complexity that represents, from a scientific-social perspective, the conservation of fragmented ecosystems immersed in diverse and unequal cultural and productive matrices, and that have been the object of the implementation of a public-private conservation strategy called Valley Conservation Landscape San Pedro River. We investigated the sociocultural processes and the meanings and uses attributed locally to the forest and its sustainability. Based on the findings, we invite to reflect on the notion of heritage weft as a concept that, in contexts similar to that of this study, can account for a series of physical and symbolic, material and immaterial aspects, which coexist and whose interrelation makes sense and value to people. In this way, we propose a look to address the management of the conservation of the austral forest, taking into account local history and the various biological, environmental, economic and social processes that have shaped the territories.

Keywords: conservation landscape; sustainability; native forest; cultural matrices; productive matrices; San Pedro river; heritage weft

\author{
Sumario \\ 1. Introducción 5. Discusión \\ 2. Aproximación conceptual $\quad$ 6. Conclusiones \\ 3. Aproximación metodológica Agradecimientos \\ 4. Resultados Referencias bibliográficas
}

En memoria de Simón Baeza Góngora, quien fuera balsero del río San Pedro y longevo habitante del bosque austral.

\title{
1. Introducción
}

La historia de los bosques nativos en Chile está estrechamente ligada a la historia de las transformaciones del paisaje y de los distintos modelos de explotación. Cada uno de estos modelos está ligado a su vez al tipo de imaginario que en distintas épocas predominó respecto al uso y el manejo de la naturaleza. La disminución de biodiversidad y hábitats naturales se ha configurado así, desde mediados del siglo xx, en una temática de interés que trasciende fronteras políticas y disciplinarias y moviliza a agentes científicos, institucionales y ciudadanos en diversas escalas a través de la conservación. Si bien en la actualidad hay ciencias que han involucrado sustantivamente los enfoques provenientes de la ecología, la biología y la economía, en lo que concierne al manejo de la naturaleza, la conservación ha debido considerar también aspectos sociales y culturales. El abordaje científico-social de este fenómeno puede desvelar simultáneamente la configuración de una diversidad de significaciones atribuidas a 
la naturaleza (según culturas y racionalidades diversas), las cuales poseen una dimensión política y varían según las determinaciones culturales e históricas en las que se gestan (Descola, 2001).

En las áreas rurales donde se llevan a cabo procesos de conservación, se entrecruzan dinámicas y diferencias socioeconómicas, sociodemográficas y territoriales. En virtud de ellas, a categorías tradicionales de conservación como los parques o las reservas nacionales, han ido sumándose categorías que las complementan, estrategias que sirven para controlar la presión sobre los entornos naturales. En el caso de Chile, las áreas de conservación incluidas dentro del Sistema Nacional de Áreas Silvestres Protegidas por el Estado (SNASPE) albergan una importante proporción de ecosistemas, pero no la totalidad (Tecklin y Catalán, 2005). Esta situación se ha convertido gradualmente en una problemática de interés también nacional e internacional, ante lo cual, en el caso particular de la región de Los Ríos, agentes de la conservación y el desarrollo han promovido la creación de una nueva categoría de área protegida denominada Paisaje de Conservación ${ }^{1}$ (PNUD-GEF, 2013). La particularidad de esta categoría consiste en la conservación de dos tipos de ecosistemas de bosque nativo: laurifolio de Los Lagos y caducifolio del sur, vulnerables y escasos a nivel nacional (Ministerio del Medio Ambiente, 2014), presentes en paisajes rurales y predios particulares. Es decir, la conservación de estos ecosistemas requiere necesariamente de la voluntad de sus propietarios y del ejercicio público-privado con clara orientación participativa y hacia la co-gestión.

Entre los años 2008 y 2013 surge el proyecto PNUD-GEF-Sirap, que busca implementar el primer Sistema Regional de Áreas Protegidas (Sirap) ${ }^{2}$ públicoprivado y que constituye como resultado el Paisaje de Conservación Valle Río San Pedro ${ }^{3}$ (en adelante, PCVRSP), la primera unidad con dicha categoría de conservación en Chile. Institucionalmente, el PCVRSP ha sido liderado en su etapa inicial por el Ministerio del Medio Ambiente y, en la actualidad, por las municipalidades de Los Lagos y Máfil a través de oficinas técnicas. Esta categoría implementada actualmente configura un tipo de dinámica sociocultural particular y refleja los efectos de los procesos que históricamente han incidido en la transformación de los territorios australes, en la degradación del bosque nativo y los hábitats naturales y en la configuración de distintos imaginarios

1. Categoría de área protegida que utiliza como referencia la categoría V Paisaje Terrestre/ Marino Protegido, propuesta por la Unión Internacional para la Conservación de la Naturaleza (UICN).

2. Según el PCVRSP, la categoría Paisaje de Conservación cumple un rol de soporte ecológico en el sistema de áreas protegidas, en tanto promueve la conservación de territorios relevantes para la biodiversidad fuera de los límites de las áreas protegidas bajo preservación e incorpora dentro de sus objetivos la provisión de servicios ecosistémicos a la población. Fuente: <www. valleriosanpedro.cl>.

3. La iniciativa PCVRSP, primero como proyecto y luego institucionalizada en ambas municipalidades, fue impulsada entre los años 2008 y 2013, en el marco del proyecto GEF Sistema Regional de Áreas Protegidas (Sirap), contando con apoyo internacional del Fondo para el Medio Ambiente Mundial (GEF) y del Programa de las Naciones Unidas para el Desarrollo (PNUD). 
de la sustentabilidad del bosque que se constituyen en el objeto de estudio de esta investigación.

Desde una perspectiva antropológica y un enfoque de ecología política (Alimonda, 2015; Leff, 2015; Santamarina, 2008), existe una dimensión de dicha estrategia que desde una óptica parcial puede pasar desapercibida y que guarda relación con el plano de lo intersubjetivo, lo imaginario y lo simbólico. ¿Qué significa el bosque nativo para los distintos actores sociales presentes en el territorio? ¿Qué rol cumplen aquellos significados (y las prácticas en las que se materializan) en la sobrevivencia de los ecosistemas entendidos como prioritarios de conservar? El presente trabajo corresponde a un estudio etnográfico realizado durante el año 2016 cuyo objetivo fue explorar los imaginarios de la sustentabilidad del bosque nativo, para así responder a la pregunta acerca de qué valores atribuidos al bosque nativo se privilegian y cuáles son subordinados u obviados, y las implicancias que ello tiene en la conservación in situ del bosque y de paso en el porvenir de la naturaleza.

\section{Aproximación conceptual}

\subsection{Imaginarios sociales de la sustentabilidad}

Abordar la configuración de imaginarios sociales en torno a la sustentabilidad de los bosques nativos, en un territorio que ha sido históricamente transformado y que hoy se sitúa como un espacio prioritario para la conservación de la biodiversidad, implica prestar atención a dos dimensiones que se interrelacionan: una sociopolítica y otra sociocultural.

Desde el punto de vista político, la primera dimensión hace referencia al proceso de instalación del paradigma de la sustentabilidad a escala global, las dimensiones desde donde ha sido abordada y la actual reflexión crítica en torno a sus cimientos, ambigüedad y alcances.

Esta dinámica se relaciona con la sustentabilidad, un concepto que emerge y ha sido permeado discursivamente tras la diseminación del desarrollo sustentable promovido por la Organización de las Naciones Unidas (ONU) a través de la publicación del Informe Brundtland en 1987. Este correspondería al nuevo lineamiento verde que llevaría desde entonces el capitalismo, en respuesta a las presiones del movimiento ambientalista que denunciaba las consecuencias del crecimiento económico posterior a la Segunda Guerra Mundial registrado en los países industrializados y las crisis que afectaban a otras latitudes del mundo (Jankilevich, 2003). En dicho informe, el desarrollo sustentable fue definido como el que permite satisfacer las necesidades de la presente generación sin comprometer la capacidad de las generaciones futuras para satisfacer las suyas integrando las dimensiones económica, ecológica y social. De esta forma, la ONU opta por una definición antropocéntrica de sustentabilidad (Beaucage, 2007; Tyrtania, 2016).

Considerando lo anterior, se entiende el desarrollo sustentable como un ideopanorama, concepto propuesto por Appadurai (1990) para referirse a 
aquellos «elementos de la visión del mundo del iluminismo que consisten en la concatenación de ideas, términos e imágenes, incluyendo libertad, bienestar, derechos, soberanía, representación y el término matriz democracia» (en Ribeiro 2011: 164). Ribeiro (2011) señala la existencia de un gran número de ideopanoramas que circulan en el interior del sistema mundial, que influyen y tienen consecuencias sobre políticas globales, como por ejemplo las del desarrollo y las de conservación. De esta forma, hace referencia a la Conferencia de Río (1992), circunstancia que describe como «un mega ritual global de integración de las elites transnacionales, [que] fue crucial para consolidar la interpretación del desarrollo sustentable como la nueva ideología/utopía del desarrollo» (ibíd.: 173). Si bien fue establecida una definición por parte de la ONU, su falta de precisión ha provocado diversos debates básicamente por la ambigüedad del concepto (Naredo, 1996; Barkin, 1998; Gudynas, 2004) y, ciertamente, por el «abismo creciente entre el concepto de desarrollo sustentable y las prácticas actualmente dominantes» (Beaucage, 2007: 44).

Desde el punto de vista sociocultural, la segunda dimensión se refiere al proceso de construcción de imaginarios sociales y su recíproco vínculo con procesos de transformación del entorno natural históricamente determinados. Esta dinámica tiene relación con el concepto de imaginario social, definido por Castoriadis (2007) como una construcción simbólica y un proceso creador permanente que permite instituir, crear y modificar las sociedades, es decir, lo que conserva unida a una sociedad es el mantenimiento conjunto de su mundo de significaciones a través de un magma cohesionante (Girola, 2012). Estas significaciones imaginarias sociales se encarnan en instituciones (de poder, económicas, familiares, etcétera) y, una vez creadas, tanto las significaciones como las instituciones se cristalizan o se solidifican, lo que Castoriadis llama un imaginario social instituido. Es este el que asegura y da sentido a la continuidad de la sociedad, lo que se entiende como realidad y la repetición de las formas que regulan la vida de los sujetos, hasta que una nueva creación de significaciones venga a modificarlos por otras formas (Castoriadis, 2001, citado en Girola, 2012). Los imaginarios sociales, que no deben ser entendidos como representaciones estáticas sino como esquemas de representaciones, se relacionan con las visiones de mundo y con la creación de imágenes con sentido que permiten acceder a la interpretación de lo social (ídem, 2012), para generar mecanismos por los cuales un orden social es normalizado y naturalizado por los individuos a través de procesos simbólicos de legitimación social (Pintos, 1995).

Con relación a la sustentabilidad, este concepto es comúnmente entendido como un atributo de las dimensiones del sistema económico de las que se desprenden efectos medibles, que mantienen o alteran la sustentabilidad económica, ecológica, ambiental, social, etcétera. En la medida en que la sustentabilidad pasa a ser apropiada por el universo discursivo de las instituciones (gubernamentales o no gubernamentales), incluso de empresas de distinto tipo, se diversifican los significados atribuidos al concepto. Es así como la noción de sustentabilidad "puede ser utilizada para significar casi cualquier cosa que uno desee, lo que constituye parte de su atractivo» (O’Connor, 2002: 27), razón por 
la cual, en palabras de Toledo (2015: 36), «del mito del desarrollo se ha pasado al mito de la sustentabilidad. Se hace entonces urgente y necesario impensar la sustentabilidad (...), es decir, intentar una desmitificación del concepto». En este sentido, Barkin (2002: 185) enfatiza que la «sustentabilidad no es simplemente un asunto del ambiente, de justicia social y de desarrollo. También se trata de la gente y de nuestra sobrevivencia como individuos y culturas».

Por su ambigüedad y los actuales alcances que posee, Leff (2010) destaca la necesidad de indagar en otras nociones de sustentabilidad en la diversidad cultural, lo que permitiría atender «la posible capacidad de respuesta desde identidades culturales situadas, que pudieran movilizar a nuevos actores sociales [dando espacio a] una democracia ambiental desde las condiciones ecológicoculturales instituidas en los imaginarios sociales» (ibíd.: 42). Tales condiciones a las que se refiere Leff, para ser halladas en los imaginarios sociales, deben ser contextualizadas históricamente de modo que revelen la recíproca relación que existe entre la construcción-transformación de imaginarios sociales y la transformación del entorno natural (y social) que ocurre de determinada manera y no de otra, lo que da cuenta de racionalidades que disputan el control de un imaginario sobre otros. Se tiene entonces que los imaginarios resultan ser siempre plurales y contingentes, por cuanto están siempre construyendo realidades, cumpliendo importantes funciones en el mundo y en las sociedades contemporáneas inmersas en el dinamismo de la globalización. De ella se desprende la proliferación de sentimientos de incertidumbre acerca del futuro del planeta por aspectos ecológicos y económicos. En este punto emerge el asunto del poder, que da cuenta de lo que Baeza (2000) ha señalado como imaginarios sociales dominantes y dominados, en referencia al monopolio o hegemonía que un imaginario puede alcanzar sobre otros. Este dominio no solo puede producirse por una sociedad sobre otra, sino entre sectores de una sociedad o a niveles intragrupales. En palabras de Baeza y Silva (2009: 31), «un imaginario social puede ser considerado como dominante solo en la medida en que sus contenidos han sido naturalizados, por lo tanto adoptados por el conjunto de la sociedad y transformados en habitus (...), y en mentalidad cuando dichos contenidos perduran por muy largo tiempo y devienen en estructuras mentales muy estáticas».

Con relación al contexto de estudio, la vulnerabilidad de los ecosistemas de bosque nativo, dada por su escasez y su presencia en predios privados, comporta observar desde una óptica biológica y ecológica el espacio biofísico en que se sitúan. Sin embargo, es pertinente prestar atención también al campo sociosimbólico que se configura en dicho contexto socioambiental y en las relaciones humano-ambientales que convierten estos espacios biofísicos en espacios cargados de múltiples actividades humanas, de historia y de imaginarios (Ther, 2012). Esto explica por qué en dichos espacios aún perviven fragmentos de bosque nativo, cuando en áreas circundantes el bosque fue explotado o convertido en praderas. Desde una perspectiva de ecología política, este tipo de procesos requiere de una reflexión centrada en las relaciones de poder y saber que envuelven a la naturaleza «y a su apropiación, transformación, produc- 
ción y control. Si la ecología a secas pretende ser ciencia, la ecología política es [entonces] análisis político en torno de la naturaleza» (Palacio, 2006: 155).

En el actual momento de reflexión sobre la naturaleza y el medioambiente y su dimensión política, por ejemplo, a través de la implementación de estrategias de conservación y desarrollo sustentable, resulta apropiado posicionar los imaginarios sociales en «la arena política, allí donde (...) se apuntalan procesos de reidentificación cultural con la naturaleza, de donde emergen nuevos actores sociales en el encuentro con la racionalidad moderna, ante la globalización y en la construcción de sociedades sustentables» (Leff, 2010: 110).

\subsection{La construcción social del paisaje: de un paisaje de degradación a un paisaje de conservación}

Abordar la noción de paisaje como relato del cambio en el entorno y en la historia implica señalar la definición de paisaje que surge a propósito del Convenio Europeo del Paisaje en el año 2000, en la que se asume su sentido territorial: «Por paisaje se entenderá cualquier parte del territorio tal como la percibe la población, cuyo carácter sea el resultado de la acción y la interacción de factores naturales y/o humanos». Esta definición sustenta sus bases en "preocupaciones ambientales y culturales, con una motivación eminentemente social y articulada en torno a tres nociones básicas: territorio, percepción y carácter» (Mata et al., 2010: 119).

$\mathrm{Al}$ situar el paisaje en una dimensión fenomenológica y al centrar la atención en que está constituido por un carácter, se desprende la dimensión histórica y cultural del concepto. El tiempo histórico es decisivo en la configuración paisajística, y se entiende así que dicho carácter «es resultado de la acción de factores naturales y humanos y de sus interrelaciones» (ibíd.). Desde esta perspectiva, se asume que el paisaje es dinámico. En este sentido, se articulan tres dimensiones en la construcción sociocultural del paisaje: el espacio físico, el espacio social o construido y el espacio simbólico (Criado, 1999), dimensiones que permiten hallar en el paisaje habitado, explotado y actualmente conservado (Costa, 2009) elementos para comprender, en el caso particular de esta investigación, la configuración de distintas maneras de significar el bosque nativo. Abordar la historicidad de este constructo nos permite reconocerle desde su condición de ser materia prima para la industria maderera durante largo tiempo hasta ser hoy motivo para definir, como sitio prioritario de conservación, el área en donde a fragmentos se distribuye.

\section{Aproximación metodológica}

Para aproximarse al objeto de estudio, se empleó un diseño metodológico cualitativo (Denzin y Lincoln, 2012) y un enfoque etnográfico (Guber, 2011; Hammersley y Atkinson, 1994). Se utilizó como estrategia metodológica el estudio de caso (Yin, 1989) y un muestreo de tipo no probabilístico e intencionado, con un muestreo por conveniencia y un muestreo en cadena de acuerdo con 
Tabla 1. Clasificación de actores y muestra

\begin{tabular}{lll}
\hline Clasificación & Descripción & Abreviación \\
\hline PCVRSP Institución & Actores que representan a la institucionalidad & Funcionario \\
\hline $\begin{array}{l}\text { Propietario de predio } \\
\text { PCVRSP }\end{array}$ & $\begin{array}{l}\text { Habitante local propietario de predio que participa } \\
\text { en el PCVRSP }\end{array}$ & Propietario \\
\hline $\begin{array}{l}\text { Habitante local ajeno } \\
\text { a PCVRSP }\end{array}$ & $\begin{array}{l}\text { Habitante local que no participa en el PCVRSP } \\
\text { y forma parte del área de estudio }\end{array}$ & Habitante \\
\hline
\end{tabular}

Fuente: elaboración propia.

las etapas del trabajo de campo realizado (Quintana, 2006). Bajo el criterio de abarcar en un sentido exploratorio a los tipos de actores locales presentes en el territorio que tienen un mayor grado de relación con los remanentes de bosque nativo, la muestra está compuesta por actores diferenciados en tres unidades de análisis (categorizados como Funcionario, Propietario y Habitante).

Como menciona Yin (1989, citado en Jiménez, 2012), el estudio de caso considera diversas variables para las cuales es necesario el acceso a diferentes

Figura 1. Localización del área de estudio en la comuna de Los Lagos (Chile)

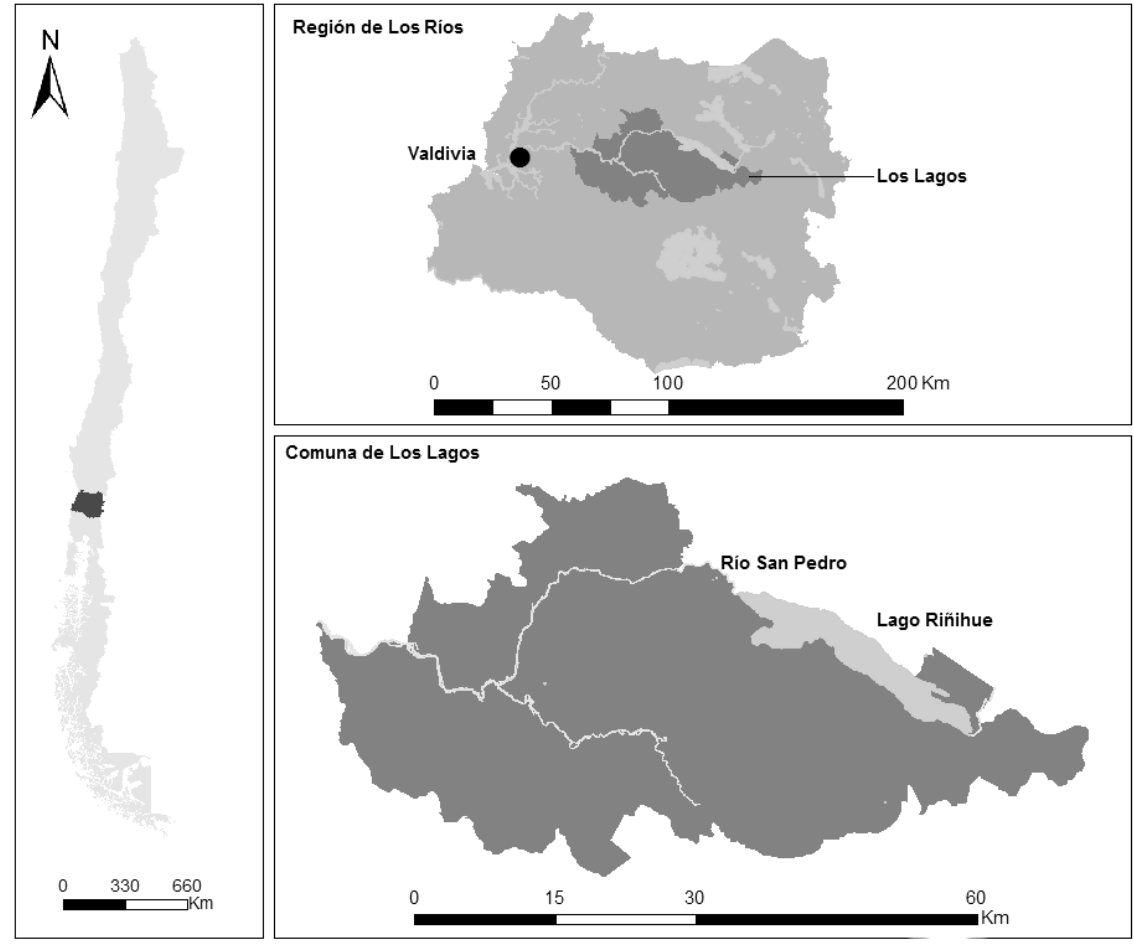

Fuente: elaboración propia. 
Figura 2. Localización del Paisaje de Conservación Valle Río San Pedro

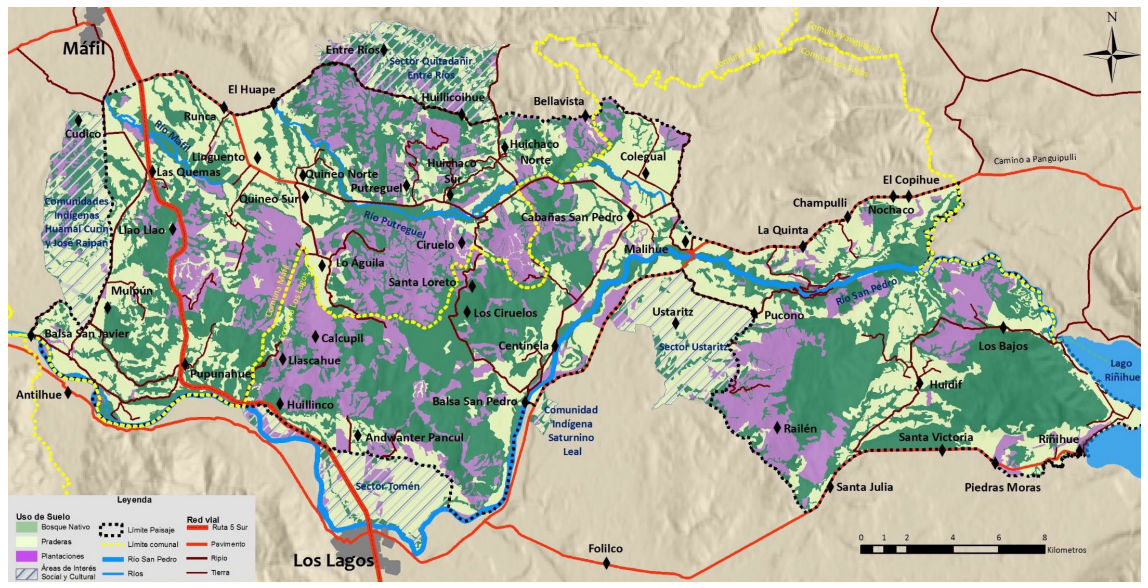

Fuente: PCVRSP.

fuentes de información. Esta investigación considera datos primarios obtenidos de entrevistas etnográficas, notas de campo, observación directa y participante (Flores, 2009). Asimismo, considera documentos relativos a la institución PCVRSP, disponibles en línea y proporcionados en instancias de participación directa (seminario, reuniones en la comuna de Los Lagos), y la revisión de documentos de carácter científico e histórico; estudios relacionados con el bosque nativo; documentos estadísticos censales, comunales y regionales; documentos de carácter normativo sobre conservación de la biodiversidad, políticas de manejo de recursos naturales y desarrollo sustentable. El tratamiento de los datos se realizó mediante análisis del discurso (Santander, 2011) y triangulación hermenéutica, a modo de entrecruzar la información y construir el corpus de resultados (Cisterna, 2005).

La muestra, definida por el criterio de saturación teórica, quedó conformada por 20 actores de los siguientes sectores de la Provincia de Valdivia: Riñihue, Desagüe Riñihue, Santa Mónica, Ustaritz, Malihue, en la comuna de Los Lagos; y de los sectores de Pishuinco y Huellelhue en la comuna de Valdivia.

Tabla 2. Muestra de estudio

\begin{tabular}{lcc}
\hline Actores & Hombre & Mujer \\
\hline PCVRSP Institución & 1 & 1 \\
Propietario de predio PCVRSP & 1 & 2 \\
Habitante local ajeno a PCVRSP & 10 & 5 \\
\hline Total & & 20 \\
\hline
\end{tabular}

Fuente: elaboración propia. 
El análisis se centró en la dotación de sentidos y significados por parte de actores locales y se indagó en aspectos identitarios y territoriales por encima de aspectos ecológicos y económicos. Los resultados fueron ordenados de la siguiente manera: 1) presentación de una síntesis de los significados y usos atribuidos al bosque, 2) comparación de los imaginarios de la sustentabilidad del bosque nativo desde una perspectiva de ecología política.

\section{Resultados}

\subsection{Significados y usos cambiantes del bosque nativo}

La información recopilada a partir de entrevistas, en concordancia con la revisión de documentos históricos relacionados con el uso del bosque nativo por la industria maderera durante la primera mitad del siglo xx y su posterior sustitución por monocultivo forestal, permite identificar un tipo de valoración de larga data entre los habitantes del valle del río San Pedro. Esta se relaciona estrechamente con procesos político-económicos ocurridos en el país, representados por los momentos de instalación de modelos económico-productivos e ideologías dominantes en el territorio, en los cuales los bosques nativos fueron considerados como recursos naturales cuya explotación era necesaria para el progreso del país.

\subsubsection{El bosque nativo como madera}

La región de Los Ríos y la zona centro sur de Chile en general estuvieron caracterizadas por tener una alta presencia de bosques nativos a orillas de los ríos y lagos, lo que, como señalan Solari et al. (2011) y Moya y Vásquez (2014), facilitó en gran medida la explotación del bosque como recurso forestal. Las dinámicas sociales y económicas que se generaban durante la primera mitad del siglo xx en torno a la explotación maderera, y por tanto en torno a la estimación del bosque como recurso forestal, influían en la valoración del río San Pedro. En este contexto, el río era también concebido como recurso para la economía, debido a su especial interrelación con el bosque, ambos valorados como fuentes de recursos y fuentes de trabajo. En dicho auge, emerge también la labor del balsero, actor social encargado de transportar en balsas, a través de los cursos fluviales y lacustres, la madera nativa para su posterior entrega y comercialización, principalmente en la ciudad de Valdivia.

Sin embargo, en el territorio que abarca la cuenca del río Valdivia, ocurrió un desastre socionatural que influyó drásticamente en las dinámicas sociales y económicas que se generaban en torno a la explotación de los bosques nativos y el uso de los ríos como medio de transporte de la materia prima y de los habitantes. El terremoto de 1960 , con sus 9,5 grados de magnitud, provocó múltiples problemas en la cuenca del río Valdivia. Uno de los más importantes fue el evento conocido como el Riñihuazo (Araya et al., 2014; Castedo, 2000; Davis y Karzulovic, 1963; Canisius, 1961), que alteró significativamente el lugar de nacimiento del río San Pedro en el lago 
Riñihue. El terremoto produjo deslizamientos de tierra, conocidos como tacos 1, 2 y 3 en este lago, y provocó la incertidumbre social entre quienes habitaban las localidades próximas a los ríos San Pedro y Calle Calle. Varios días después del seísmo, los deslizamientos fueron visibles y percibidos por los habitantes de estas tierras. El desagüe del lago Riñihue por el río San Pedro se había obstruido y amenazaba con inundar los pueblos que lo bordeaban. Como respuesta, se generó un despliegue excepcional de personas en una acción colectiva llamada «la epopeya del Riñihue», también conocida como el Riñihuazo, entre los días 25 de mayo y 10 de agosto de 1960 (ENDESA, 1962). Producto de toda esta situación, se suscitaron otro tipo de problemas al borde y en la navegabilidad del río San Pedro, ya que "se generaron muchos rápidos en el río, que era imposible subir. Hasta ahí llegó el tema de la navegación» (Habitante 8, 2015).

\subsubsection{El terremoto de 1960, la expansión del monocultivo y la distinción de lo nativo y lo exótico}

Los habitantes señalan el cambio que sobrevino en el territorio, el paisaje y el bosque. Después del terremoto, el auge maderero en la zona comienza su descenso y los bosques dejan de ser un recurso valioso en términos económicos. Las grandes extensiones de tierra donde antiguamente había bosque nativo comenzaron a ser reforestadas con monocultivo, principalmente eucalipto y pino radiata. Esta transformación económica de los dueños de los fundos estuvo incentivada por el Estado mediante el D. L. 701 de 1974, que subsidiaba gran parte de estas plantaciones. "Los ricos en muchas partes pusieron pino y eucalipto porque estaba limpio, y el bosque nativo había desaparecido antes» (Habitante 11, 2016). En la medida en que resultaba evidente, los habitantes comenzaban a distinguir los tipos de árboles, a diferenciar entre lo nativo y lo exótico. Identificaban los procesos de sustitución del bosque nativo y de reforestación con plantaciones exóticas: «Miraba ahí de camino de Paillaco a Valdivia, hay una parte antes de llegar a Las Alturas, joiga pero un tremendo cerro de puro plantío no más!, pura reforestación. Nada de nativo» (Habitante $11,2016)$.

La plantación exótica provoca distintas reacciones entre los habitantes y resulta común una valoración negativa expresada en ideas: «Estamos invadidos por forestales» (Habitante 10, 2015) o "Es muy invasivo» (Propietario 2, 2016). No obstante, también actúa como una referencia por oposición para brindarle otros valores al bosque nativo: «Esta cuestión de la reforestación echa a perder el ambiente, el aire. El árbol nativo, no, porque la función del árbol nativo, la hoja, es purificar los aires; así que es un favor para nosotros, para la comunidad» (Habitante 11, 2016).

El bosque que todavía queda empieza a ser valorado de forma diferente a como lo era en el pasado reciente. En el discurso de los habitantes, se reconocen transiciones, así como un proceso paulatino de "concienciación" respecto al uso predominante al que fue reducido el bosque nativo durante gran parte del siglo pasado y al desaprovechamiento en el acto de tirar o quemar lo que no valía como madera. Es valorado el bosque actual y también es resignificado el 
bosque explotado, el bosque que ya no existe, aquel que se recrea como una imagen en la memoria de quien, por ejemplo, trabajó en el ámbito maderero. Sobre este bosque se reflexiona: «En ese tiempo, me hubiera dado cuenta de lo que conozco ahora... Se cometía un daño y el que aprovechaba todo era el dueño del fundo, los ricos, y el pobre, puro trabajo y poca la utilidad para el trabajador» (Habitante 11, 2016).

Asimismo, en la memoria de la familia se recrean los otros usos dados al bosque relacionados con la recolección de «digüeñes, el mosqueto, el maqui, la murra, el chupón, la murta, toda esa clase de frutos de por aquí, por Riñihue» (Habitante 12, 2016), con los cuales se elaboraban productos como «la chicha de maqui, una chicha más buena que hacíamos. Las antiguas hacían chicha de maqui, y se comía también» (Habitante 13, 2016).

Para los habitantes, esta diversidad de usos pasados del bosque nativo son resignificados en el momento de comparar los estilos de vida antiguos y el actual, sobre todo con relación a la alimentación, la salud y el bienestar. Hoy la relación, o más bien la oposición entre lo nativo y lo exótico, permea lo que se entiende también por naturaleza: «La cuestión de tanto plantío de pino... ¡Y varias clases de pino! Yo no conozco toda la variedad de pino que hay. Hay eucalipto, y eso va en perjuicio de la naturaleza» (Habitante 11, 2016).

\subsubsection{Proteger el bosque que queda}

Desde el momento en que los bosques nativos explotados dejan al descubierto los cerros, las quebradas y, posteriormente, se produce la introducción de nuevas especies de árboles, el bosque nativo comienza a ser resignificado como parte de la naturaleza, mientras que lo exótico es lo "artificial» (Habitante 4, 2015). En concordancia con esto, aquello que habita en la naturaleza en formas vegetales, animales o inanimadas se va enfrentando a la histórica y constante transformación del paisaje. Pero los bosques esta vez empiezan a ser valorados ya no solo como recurso para el consumo actual, sino para las futuras generaciones de humanos y de otras especies. El bosque se convierte en un "patrimonio, lo nuestro», que es necesario proteger y heredar. Se va configurando así una comprensión del bosque como patrimonio, el cual debe mantenerse "para que la gente joven vea lo que había, que alguien recuerde. Así como estamos nosotros, va a haber otra persona que va a contar algo parecido" (Habitante 13, 2016).

La significación del bosque como patrimonio adquiere matices según los motivos por los que los habitantes lo heredarían. Por ejemplo, desde la perspectiva del paisaje, «tenemos que cuidar los pocos lugares como estos que nos van quedando (...), no podemos dejar que ellos [los bosques y el lago Riñihue] se mueran» (Habitante 2, 2015). En un sentido similar, debe mantenerse en tanto es hábitat de especies diversas, razón por la cual hay que proteger «la naturaleza primero, el bosque nativo que hay en su entorno, las nalcas, el pudú» (Habitante 7, 2015), y otras especies nativas que conviven en el territorio, aves como el chucao y el martín pescador, mamíferos como el puma, el huillín y el monito del monte, peces como el tollo de agua dulce, entre otros. 
La relación entre el bosque nativo y el río continúa en este tipo de significación. Es una relación que se anhela proteger también como patrimonio, significada por los habitantes de diversas formas con la ampliación de los matices mencionados previamente. «Recordemos la cantidad de fósiles que hay a orilla del río... Y a lo largo de todo el río hay fósiles» (Habitante 7, 2015). Una de las manifestaciones del valor patrimonial de la relación entre el bosque nativo y el río San Pedro se configura en el uso y la resignificación del patrimonio paleontológico correspondiente a improntas foliares fosilizadas, hojas de bosques nativos antiguos y/o extintos presentes en la ribera del río San Pedro, que dan cuenta de la prolongada existencia del bosque nativo . Este patrimonio se encuentra protegido por el Estado de Chile mediante la ley $\mathrm{n}^{\circ} 17.288$ de Monumentos Nacionales.

Asimismo, el bosque y el agua emergen de forma simbiótica cuando los habitantes se explican la escasez hídrica por la sustitución y la reforestación con plantaciones exóticas. En dicha relación, es posible identificar cómo los habitantes sitúan en polos opuestos lo nativo y lo exótico, donde lo exótico significa sequía y lo nativo significa agua: «Aquí antes era todo nativo, se echó todo abajo y se vinieron con esto. Esto nos está destruyendo» (Habitante 1, 2015). El agua como elemento patrimonial se posiciona como fundamental en la reproducción de la vida: «El gran patrimonio que uno le puede dejar a sus hijos y a todas las generaciones que puedan venir es el agua. Todos sabemos que el agua es vida. Y si alguien la interviene, ¿qué patrimonio les podemos dejar?» (Habitante 10, 2015).

\subsection{El bosque nativo como condición para la reproducción de la vida biológica $y$ cultural}

Algunos de los usos y significados cambiantes del bosque nativo que han sido identificados y descritos, entendidos de acuerdo con el contexto histórico en que son producidos por los habitantes, reflejan los cambios sociales, económicos, geológicos, geográficos, ambientales y culturales del territorio. Los diversos usos y significados sitúan hoy al bosque nativo como un bien cuyo valor ha trascendido, desde que fue considerado como un obstáculo para la expansión de la frontera agrícola o reducido a madera para exportación durante el auge maderero en la primera mitad del siglo xx.

Así, el bosque nativo ha comenzado a ser valorado también desde una relación recíproca que los habitantes han identificado entre el bosque nativo y el agua, en un contexto donde los paisajes son paulatinamente diversificados por la acción antrópica creando un mosaico de praderas, bosque nativo y plantaciones exóticas. En este contexto, la escasez de agua está presente, «quizás no tanto en el paisaje, pero sí en los discursos de la gente con la que tú trabajas. Hoy día los acentos están puestos en recuperar el bosque porque hay que recuperar el agua» (Funcionario 1, 2016). Así es como el bosque nativo es también agua, y su interrelación es usada por los habitantes para reproducir sus actuales condiciones de vida, puesto que paulatinamente han establecido la conexión «de que, efectivamente, si no luchan por el bosque, no tienen posibilidades de 
asegurar el agua, y está también muy instalado en el colectivo que no solo es para ellos, sino para sus hijos» (Funcionario 1, 2016).

Al mismo tiempo, entre los habitantes ha adquirido relevancia una problemática en torno al río San Pedro, porque en este fue iniciada - aunque se encuentra detenida actualmente- la construcción de la central hidroeléctrica San Pedro. La represa ha sido objeto de conflictos locales y cuestionamientos por los cambios que producirá en el ambiente, en la biodiversidad, en las prácticas y en los usos dados a los ecosistemas (bosques y ríos), además de involucrar a propietarios y habitantes del PCVRSP. Desde 2007, año en que la empresa presenta el proyecto a evaluación ambiental, se ha mantenido la problemática hasta la actualidad dado que el proyecto, tras varios intentos fallidos, reingresó en diciembre de 2018 en el Sistema de Evaluación de Impacto Ambiental (SEIA) (BioBio Chile, 2018; Palma, 2019), mediante el cual se aprueban o rechazan proyectos de este tipo. Los funcionarios son conscientes de dicha problemática:

El tema de la represa significó algo. Siempre, cuando los laguinos hablan de este paisaje y tratan de rememorar cuál ha sido uno de los hitos más importantes de la lucha que han tenido por proteger su territorio, inmediatamente se acuerdan del tema de la represa. Y de cómo todos tuvieron que salir a la calle a defenderlo. Es bien transversal. Yo creo que es como uno de los temas más sentidos. (Funcionario 1, 2016)

\subsubsection{El bosque como entramado}

En este sentido, se puede decir que en el área de estudio los actores locales entrevistados han reconocido y destacado la existencia de un entramado patrimonial, precisamente porque en él existen procesos biológicos, ambientales, sociales, culturales y económicos que hallan su nicho de producción en el bosque y que se interrelacionan mediante los sentidos y las prácticas humanas. La puesta en valor y la reproducción de este entramado, así como la revitalización de las prácticas sustentables (preexistentes o asumidas) de

Figura 3. Representación de los usos y significados cambiantes del bosque nativo, según los discursos de los habitantes del valle del río San Pedro

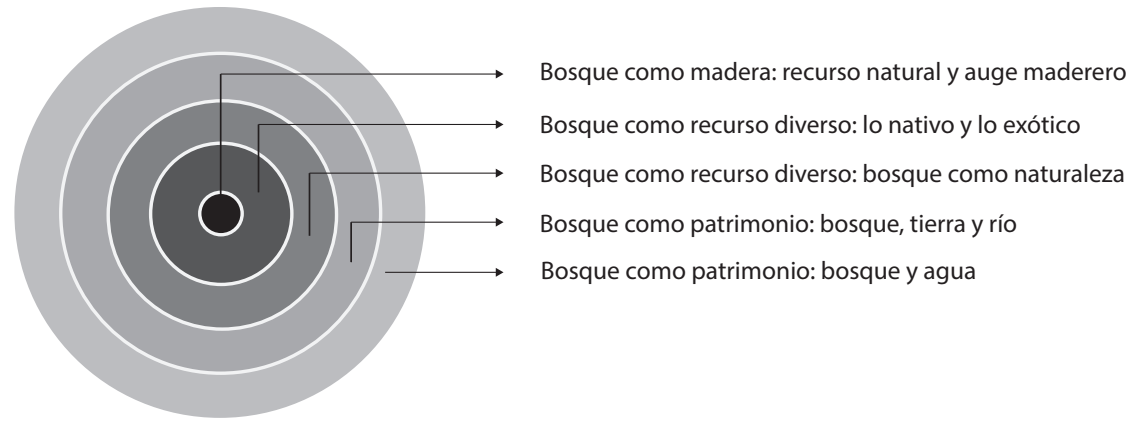

Fuente: elaboración propia. 
los habitantes, permiten o facilitan la conservación y la sobrevivencia de los ecosistemas de bosque.

No obstante, la noción de patrimonialidad no proviene del bosque en tanto objeto, sino de los sujetos y el proceso de asignación de valores y la apropiación cultural del bosque, lo que viene a formar parte del patrimonio a los habitantes mediante su selección y atribución de significados y usos del bosque, en sintonía con las capacidades que este posee para proveerlas. La figura 3 sintetiza visualmente las interrelaciones establecidas entre los elementos del bosque nativo y el espacio en el que se inserta, donde cada círculo representa un valor del bosque que amplía el anterior, de modo que pasa de ser valorado como unidad (madera) a serlo como parte de un entramado humanos-bosquebiodiversidad-tierra-río-fósiles-agua.

\subsection{Análisis de los imaginarios de la sustentabilidad del bosque nativo}

\subsubsection{Sustentabilidad como principio y acción}

A partir del análisis de la teoría y de los datos obtenidos, en esta investigación se entiende la sustentabilidad como principio organizador de las relaciones humano-ambientales y, a la vez, se constituye en acción social. En los discursos de los habitantes del valle del río San Pedro, este concepto adquiere matices: mientras que algunos aspectos se potencian por parte de las instituciones (el valor económico-ecológico de la conservación de los bosques y la biodiversidad en dinámicas productivas), otros aspectos quedan fuera de lo cuantificable (el valor patrimonial del bosque, del agua o su interrelación, o del patrimonio paleontológico presente en el paisaje de conservación) y, por ende, fuera de lo asistido institucionalmente, en tanto son aspectos que no pueden ser (o no buscan ser) traducidos a un valor de mercado.

La crisis ambiental se incorpora a las políticas que involucran el establecimiento de áreas protegidas o sitios prioritarios para la conservación, lo que se traduce en instrumentos ecopolíticos (Giminiani et al., 2016). Al reconocerse el valle del río San Pedro como un sitio prioritario para la conservación, es decir, un lugar remapeado por las tipologías globales de la conservación (West et al., 2006), cabe preguntarse cómo los sujetos usan y hacen significativo su entorno natural. Estos usos y significados del bosque han cambiado a través del tiempo en el territorio, de acuerdo con condiciones - tanto sociales, políticas, económicas como naturales - que por un largo período en la historia privilegiaron el valor de la madera por encima de otros múltiples valores que simultáneamente le han sido asignados. Actualmente, los significados del bosque nativo identificados reflejan una variedad de maneras de comprender la realidad social pasada y actual del territorio, que transitan desde una realidad social en la que predominaba un imaginario del bosque como recurso inagotable debido a su abundancia hasta una realidad en la que existe un imaginario del bosque amenazado que requiere de la concienciación de la sociedad para sobrevivir. Este último imaginario, fuertemente fomentado por el discurso institucional 
ambiental, se constituye en dominante en tanto refleja el continuo valor del bosque nativo como recurso, pero esta vez bajo una condición de riesgo.

\subsubsection{Imaginarios dominados: el bosque como sustento, el bosque como patrimonio} Sin embargo, los remanentes presentes en el territorio de estudio se hallan en tal estado porque tienen un significado para quienes resultan ser sus propietarios, considerando que se trata de bosques nativos no contenidos en áreas naturales protegidas por el Estado, sino que se sitúan en predios privados, en terrenos que además son productivos.

Estos significados son diversos. El bosque es materia prima, es madera, es leña, es forraje para los animales, es hábitat para la biodiversidad, es producción de agua, es lugar para la recolección de alimentos, es lugar para la inspiración artística, es lugar para la recreación, es herencia, es parte del paisaje. Un paisaje que además posee flora fosilizada de bosques nativos antiguos, de aproximadamente 23 millones de años (SERNAGEOMIN, 2003). Algunas de estas valoraciones son intraducibles en valores económicos (Leff, 2003), por tanto, escaparían del régimen de naturaleza capitalista (Gudynas, 2004) según el cual el bosque adquiere valor de mercado como principal mecanismo a partir de cuya misma lógica se asegura su conservación.

¿Qué ocurre con los elementos del paisaje y del mismo bosque que no entran en dinámicas de mercado? Este interrogante constituye un intento de ampliar los factores tradicionales con los que la sustentabilidad es medida según las lógicas del desarrollo, del mercado, de la racionalidad económica desde donde se producen las políticas (factores económico y ecológico), con la incorporación de aspectos que requieren necesariamente de un abordaje cualitativo. En esta investigación, dichos factores han sido denominados «factor territorial» y «factor simbólico» de la sustentabilidad de los bosques nativos (figura 4). Ambos dan cuenta de cómo determinados factores de la sustentabilidad (económico y ecológico) en el territorio se enfatizan por parte del discurso del desarrollo sustentable y pueden obviar simultáneamente otros factores y, con ello, tanto aspectos materiales como simbólicos del bosque.

Los significados y usos del bosque permiten identificar al menos dos imaginarios sociales considerados como dominados: un imaginario del bosque como sustento de prácticas y aspectos socioculturales establecidos entre sujetos y naturaleza, desde los cuales se configuran los sentidos de pertenencia y arraigo territorial y las relaciones humano-ambientales entre habitantes y remanentes de bosque nativo; y un imaginario del bosque como patrimonio, ya que del bosque nativo no solo se pueden obtener productos que van en beneficio económico de las sociedades, o en beneficio de las especies biológicas, también hay interrelaciones sociosimbólicas y socioambientales que son significativas para los habitantes, intraducibles a un valor de mercado.

Estas interrelaciones son todas construcciones socioculturales que significan al bosque nativo y amplían su valor económico, que por mucho tiempo fue hegemónico en los territorios del sur del país. Cabe destacar el significado que adquieren los yacimientos fosilíferos dentro del territorio de estudio, 
Figura 4. Representación de la ampliación de los factores relacionados con la sustentabilidad del bosque nativo en el valle del río San Pedro, de acuerdo con prácticas de sus propietarios y de los habitantes, y de acuerdo con los significados diversos del bosque

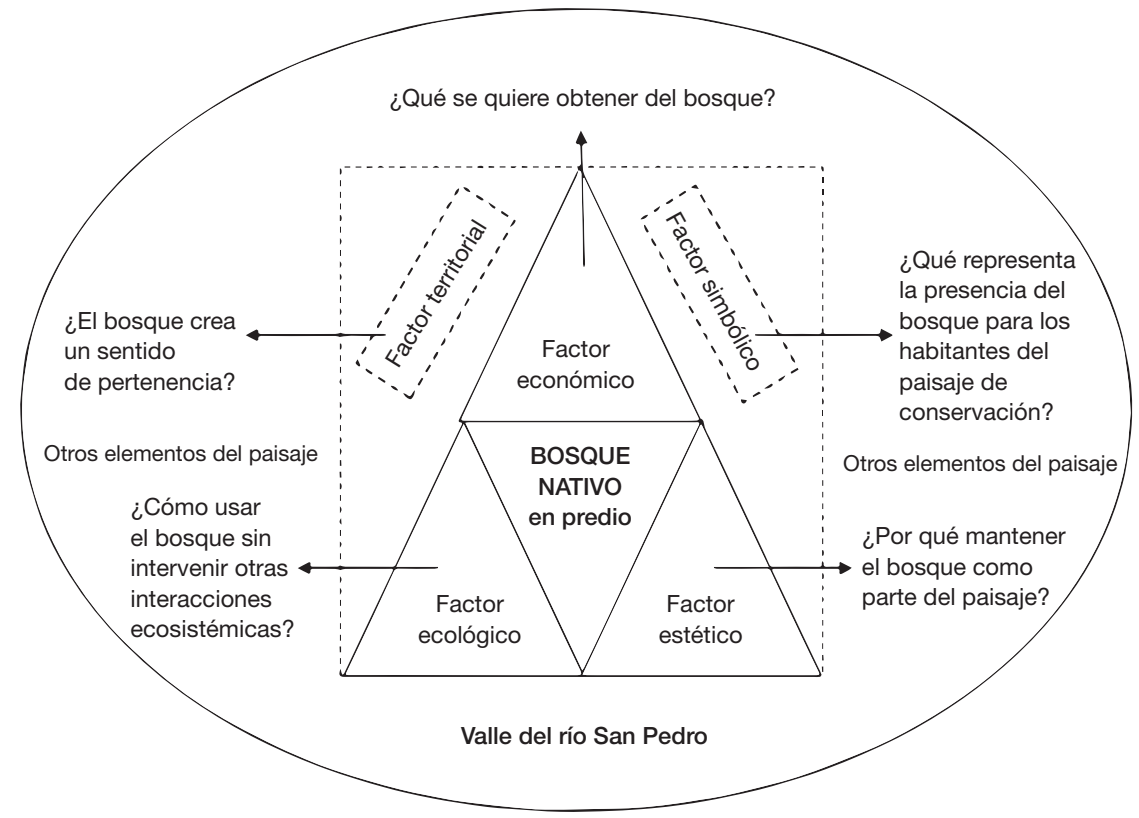

Fuente: elaboración propia.

como elemento natural presente en el paisaje de conservación. Este añade otra dimensión a aquello presente en los ecosistemas de bosque nativo que se busca proteger a través de la estrategia de conservación. Se configura como un elemento que invita a reflexionar acerca de la construcción social del patrimonio $y$, en particular, acerca de los valores que son potenciados por los lineamientos técnico-científicos del desarrollo sustentable entre las poblaciones locales. Además, cabe considerar el énfasis que le atribuyen algunos habitantes que lo conocen: «Uno va como un pasito más adelante, por ejemplo, comparado con las unidades pilotos nuevas [del paisaje de consevación], entonces uno ya pasó por la etapa de proteger el bosque, protección y conservación, todo eso. Pero es que igual uno se da cuenta de que acá hay algo que es súper interesante, igual. Es que [los fósiles] no los podemos olvidar» (Propietario 2, 2016). Desde estos dos imaginarios sociales, el bosque como sustento y como patrimonio, se pueden comprender y potenciar prácticas de sustentabilidad de bosques inmersos en matrices productivas y también de conservación, considerando que:

Lo «social» [suele quedar] subsumido dentro de lo «ecológico», es decir, mistificado, al reducirse a un elemento, variable o un factor más en la búsqueda de soluciones técnicas a la relación negativa entre seres humanos y seres vivos. 
Nunca se plantea cómo las relaciones entre los seres humanos, que pueden ser relaciones de poder, competencia, colaboración, sumisión, explotación, etc., es decir, relaciones políticas, afectan y son afectadas por las relaciones con la naturaleza. (Toledo, 2015: 40)

Ambos imaginarios de la sustentabilidad del bosque nativo permiten comprender los múltiples sentidos desde donde los habitantes le atribuyen valores. Emerge así la necesidad de repensar, a través de dichos significados, cómo ha ido configurándose la indisociabilidad del bosque y el río, del bosque y el agua, del bosque y la diversidad de formas de vida que co-habitan en el valle del río San Pedro.

\section{Discusión}

\subsection{El paisaje de conservación como conjunto de procesos y elementos indisociables}

Los imaginarios del bosque como sustento y como patrimonio ponen en relieve una profunda valoración de los ecosistemas que sobreviven en el PCVRSP. Esta valoración se fundamenta en aspectos culturales, como el arraigo territorial, la identidad local, la memoria y la confianza acerca de que el bosque asegurará algunas de las condiciones básicas para la existencia humana, como lo es la disponibilidad de agua y de elementos naturales cuyo uso sustentable beneficia a los habitantes y al bosque. Asimismo, se fundamenta en aspectos biológicos y ecológicos, en tanto el bosque asegurará algunas condiciones vitales para la biodiversidad: ser hábitat, corredor biológico y refugio de especies vulnerables. Así pues, se vuelve necesario que las miradas institucionales de la sustentabilidad atiendan este tipo de construcción sociocultural (la valoración local simbólica y material del bosque) para dar pie a una conservación de la diversidad biológica que no puede desligarse de la diversidad sociocultural.

A ello se suma la necesidad de atender los límites difusos donde comienza y donde termina el bosque nativo. En las prácticas de conservación y en la política pública, ¿se puede separar el bosque del agua?, ¿los bosques ribereños, del río San Pedro?, ¿los bosques actuales, de los yacimientos fosilíferos de bosques antiguos?, ¿'os bosques, del paisaje?, ¿’los bosques, del territorio y de la identidad local? Esta serie de preguntas, desde el análisis de los discursos de los entrevistados y de prácticas observadas en esta investigación, tiene como respuesta una negativa. No se puede separar el bosque de los otros elementos con los que convive, ni se puede separar la existencia humana de la existencia del bosque. Esto sería reflejo de un imaginario local del bosque como entramado patrimonial: una trama de significaciones que entrelaza desde lo simbólico al bosque nativo con otros elementos de la naturaleza. No obstante, esta construcción simbólica puede ser entendida como el reflejo de lo entrelazados que están materialmente el bosque, el agua, la fauna y la flora, el río San Pedro y las personas y familias que han posibilitado que estos ecosistemas fragmentados sobrevivan hasta hoy. 
Considerar las significaciones locales no solo fortalecerá las voluntades e intereses de conservación que los propietarios y habitantes han adoptado dentro del paisaje de conservación, sino que enriquecerá las miradas institucionales de la sustentabilidad a escala local y bicomunal, que es donde se materializa esta estrategia.

\subsection{Alcances de un enfoque de entramado patrimonial en la gestión de la conservación}

Como base de la actual gestión ambiental institucional, la sustentabilidad ha sido nutrida de teorías, conocimientos y métodos, así como de un conjunto de ideologías, instituciones y discursos del ambientalismo, teorías ecológicas, etcétera, en el marco de una modernidad reflexiva. No obstante, según Leff (2010: 110), ante las dudas que han generado las formas tradicionales para alcanzar el desarrollo sustentable, a través de un enfoque de ecología política, se ha abierto un espacio a "la indagatoria sobre los imaginarios sociales para intentar saber si allí se han sedimentado principios y valores de los que pudieran derivarse respuestas sustantivas». Esto implica que la reapropiación social de la naturaleza posee una dimensión política. Asimismo, tiene una dimensión epistemológica en cuanto se trata de una reapropiación social de la naturaleza desde visiones de mundo y sentidos existenciales diversos.

De lo anterior se desprende el alcance notablemente relevante, para la gestión y la conservación de los ecosistemas de bosque nativo, de atender y comprender los significados, las visiones y las valoraciones de la naturaleza que poseen los sujetos que conviven cotidianamente con ella (en referencia a otros contextos rurales similares al área de estudio, donde se hallan remanentes de bosque nativo). Esto correspondería a la construcción de un espacio en el que diversos y nuevos saberes y racionalidades sean capaces de aprehender la complejidad ambiental (Leff, 2003) complementando las formas de conocimiento desde las que las ciencias, las teorías del desarrollo y la racionalidad económica describen la naturaleza y la problemática ambiental. De esta manera, la hiperpolisemia del concepto de sustentabilidad puede configurarse en un campo para reflexionar y comprender las relaciones que los grupos sociales establecen con su entorno natural, y ya no solo ser diseminada como un criterio desde una posición unidireccional por parte de quienes producen las políticas públicas relacionadas con la conservación.

La diversidad de significados que posee el bosque permite comprender las transformaciones de las representaciones de la realidad que históricamente han sido construidas y naturalizadas, que han dado paso a un estado de degradación, escasez y conservación del bosque nativo y que han reflejado a su vez el tránsito hacia una valoración del bosque como entramado patrimonial. Asimismo, dicha diversidad da cuenta de las maneras de comprender su sustentabilidad por parte de los habitantes, y «confirma, como lo señaló Leonardo Tyrtania (...), que "la sustentabilidad es de quien la trabaja", y no de quienes solo la postulan, pregonan, analizan, promueven, pervierten o presumen» (Toledo, 2015: 52). 
Un imaginario del bosque como entramado patrimonial refleja entonces el conjunto de elementos naturales que materialmente coexisten y que simbólicamente son valorados por las personas también de forma imbricada. Cuando se habla del bosque, los entrevistados se refieren a muchos otros aspectos: agua, río San Pedro, animales, alimentos, flora, sustento, territorio, paisaje, fósiles, patrimonio. Es importante destacar entonces que la conservación no solo debe proteger el bosque o conjunto de especies forestales como unidad, desligada de las interrelaciones con otros elementos del ecosistema. La idea es promover que se aseguren las condiciones para que se conserve el hábitat y, en este caso, también se mantengan los valores y las prácticas sustentables de sus propietarios y habitantes.

\section{Conclusiones}

Respecto al problema antropológico abordado en este artículo, es posible resumir que ha sido el de la diversidad de significados asignados al bosque nativo, en un contexto biofísico, histórico y sociocultural en el que confluyen intereses de tipo económico y cultural heterogéneos. En dicha diversidad, fue posible identificar relatos, imaginarios y prácticas que cruzan contenidos globales y locales, lo que ha permitido demostrar que los desafíos de la sustentabilidad no se reducen únicamente a reconocer la diversidad de imaginarios circulando en torno a un mismo espacio, sino que implican la construcción de nuevas epistemologías que den cuenta de la pluralidad de intereses, significaciones y saberes desde un marco de reconocimiento y legitimidad de dicha diversidad. De acuerdo con los resultados obtenidos, es posible plantear que para lograr una sustentabilidad de los bosques es necesario prestar atención también a los aspectos sociales y culturales de la conservación de la naturaleza, por ejemplo, involucrando la pluralidad de intereses, conocimientos y saberes locales, a modo de complementar los conocimientos técnicos y científicos. Dichos aspectos deben ser sistematizados en el marco de la construcción de un campo de reflexión y conocimientos, nutrido por la confluencia de saberes y prácticas diversas. De esta forma, será posible encauzar una sustentabilidad cultural y territorialmente pertinente de los remanentes de bosques que aún sobreviven en el valle del río San Pedro, dentro de un marco donde la diversidad y la pluralidad de significados del bosque nativo y de la naturaleza, como aquellos identificados en este estudio, puedan ser reconocidas.

Por último, este artículo invita a reflexionar acerca de la noción de entramado patrimonial como concepto que, en contextos similares al de este estudio, puede dar cuenta de una serie de aspectos físicos y simbólicos, materiales e inmateriales, que conviven y cuya interrelación tiene sentido y valor para las personas. Se trata de una configuración de aspectos imbricados que, al ser valorados de forma individual, pueden tensionar y romper la serie de vínculos con los otros elementos que conforman esta trama. En este caso, el río San Pedro está conectado a la flora y la fauna que habitan en él y alrededor de él; los yaci- 
mientos fosilíferos están unidos al curso de sus aguas; el bosque ribereño está entrelazado a los fósiles de los bosques antiguos y es testimonio de su constante presencia; el agua emerge donde hay bosque nativo; la fauna reaparece en los ecosistemas conservados en torno al río, y todo lo anterior se encuentra en la historia vivida y relatada por sus habitantes. La visión de la categoría de Paisaje de Conservación responde a esta mirada de territorio como un todo, y su modelo de gestión permite que este entramado patrimonial que emerge desde los significados y las prácticas de los habitantes se pueda reproducir in situ y en el tiempo. En conclusión, se considera que en aquellos aspectos pueden estar sedimentados valiosos saberes y particulares formas de relacionarse con la naturaleza, vitales de atender a la hora de intentar prolongar la existencia del bosque nativo. Se trataría de aportes clave en la búsqueda de bienestar para la biodiversidad y la sociedad y, sobre todo, para poblaciones que en paisajes como los del sur de Chile, nutridos de ríos, lagos y bosques, fundamentan sus modos de vida y sus formas de ser en el mundo.

\section{Agradecimientos}

Este artículo presenta los resultados de la investigación realizada por Francisca Vergara, titulada «Bosques australes y sustentabilidad: una aproximación etnográfica a los significados del bosque nativo en el valle del río San Pedro, Región de Los Ríos" (tesis de grado), enmarcada en el seminario de título del programa de Antropología, mención Antropología Sociocultural, de la Universidad de Concepción, Chile (2016). La investigación se desarrolló bajo el alero del proyecto FONDART No 80136 «Relatos de paisaje y toponimia entre el lago Riñihue y Valdivia», ejecutado por ONG POLOC. Las autoras agradecen a ONG POLOC, al Paisaje de Conservación Valle Río San Pedro y a las y los habitantes de Los Lagos, Riñihue y Valdivia que participaron en esta investigación por la posibilidad de conocer sus experiencias de vida, y tienen un agradecimiento especial para Patricio Contreras, Natalia Campos y Francisco Torres por el valioso apoyo y la colaboración brindada.

\section{Referencias bibliográficas}

Alimonda, H. (2015). «Ecología política latinoamericana y pensamiento crítico: vanguardias arraigadas». Desenvolvimento e Meio Ambiente, 35, 161-168. <https://doi.org/10.5380/dma.v35i0.44557>.

Appadurai, A. (1990). «Disjuncture and difference in the global cultural economy». Theory, Culture \& Society, 7, 295-310. $<$ https://doi.org/10.1177/026327690007002017>.

Araya, C.; Cisternas, M.; González, F. (2014). «Evolución morfológica del principal deslizamiento del 'Riñihuazo', generado por el terremoto de 1960». VIII SLAGF, Simposio Latinoamericano de Geografía Física, IV SIAGF, Simposio Iberoamericano de Geografía Física.

BAezA, M. (2000). Los caminos invisibles de la realidad social. Chile: Sociedad Hoy RiL Editores. 
Baeza, M.; Silva, G. (2009). «Imaginarios sociales del Otro: el personaje del forastero en Chile (de 1845 a nuestros días)». Sociedad Hoy, 17, 29-38.

Barkin, D. (1998). Riqueza, pobreza y desarrollo sustentable. México: Editorial Jus y Centro de Ecología y Desarrollo.

- (2002). «El desarrollo autónomo: un camino a la sostenibilidad». En: Alimonda, H. (ed.). Ecología política: naturaleza, sociedad y utopia, 169-202. Buenos Aires: CLACSO.

Beaucage, P. (2007). «El desarrollo sustentable: crítica del discurso y de las prácticas». En: Ávila, R. (coord.). Estudios del Hombre, 22, 41-70.

BioBio Chile (2018). Critican reactivación de proyecto Central Hidroeléctrica San Pedro en Los Ríos. [Comunicado de prensa]. Recuperado de <https://www.biobiochile.cl/ noticias/nacional/region-de-los-rios/2018/12/03/critican-reactivacion-de-proyectocentral-hidroelectrica-san-pedro-en-los-rios.shtml>.

Campos-Medina, J.; Vergara-Pinto, F.; Echeverría, A.; Contreras, P.; Abarzúa, A. (2018). «Resignificación del patrimonio paleontológico presente en el río San Pedro (Cuenca del río Valdivia)». PASOS Revista de Turismo y Patrimonio Cultural, 16 (3), 655-670. <https://doi.org/10.25145/j.pasos.2018.16.047>.

Canisius, P. (1961). "Informe sobre los resultados de las investigaciones hechas por la comisión de expertos alemanes enviada a Chile después del terremoto de 1960». Universidad Austral de Chile.

Castedo, L. (2000). Hazaña del Riñihue. El terremoto de 1960 y la resurrección de Valdivia. Crónica de un episodio ejemplar en la historia de Chile. Santiago de Chile: Editorial Sudamericana.

Castoriadis, C. (2007). La institución imaginaria de la sociedad. Buenos Aires: Tusquets Editores.

Cisterna, F. (2005). «Categorización y triangulación como procesos de validación del conocimiento en investigación cualitativa». Theoria, 14 (1), 66-71.

Contreras, P.; Concha, R.; Correa, M.; Guerrero, I.; Vergara, F. (2016). Relatos de paisaje y toponimia en el valle de los ríos San Pedro y Calle Calle. Santiago de Chile: Ceibo Ediciones.

Costa, R. (2009). "La caza, la roza y el bosque: sobre los significados de la diversidad arqueológica en la subcuenca del río Santo Cristo (noroeste de rio Grande do Sul, Brasil)». Arqueología Suramericana, 5 (1), 21-41.

Criado, F. (1999). Del terreno al espacio. Planteamientos y perspectivas para la arqueología del paisaje. Grupo de investigación en Arqueología del Paisaje. Universidad de Santiago de Compostela.

Davis, S.; Karzulovíc, J. (1963). «Landslides at Lago Riñihue, Chile». Bulletin of the Seismological Soclety of America, 53 (6), 1403-1414.

Denzin, N.; Lincoln, Y. (2012) (comps.). «El campo de la investigación cualitativa». Manual de investigación cualitativa, I. Gedisa.

Descola, P. (2001). «Construyendo naturaleza, ecología simbólica y práctica social». En: Descola, P.; PÁlsson, G. (comps). Naturaleza y sociedad, perspectivas antropológicas, 101-123. México: Siglo XXI Editores.

ENDESA (1962). Sistema Hidroeléctrico Pullinque. Empresa Editora Zig-Zag S.A. Recuperado de: <http://www.memoriachilena.cl/archivos2/pdfs/MC0037322.pdf>.

Flores, R. (2009). Observando observadores: una introducción a las técnicas cualitativas de investigación social. Santiago de Chile: Ediciones Universidad Católica de Chile. 
Giminiani, P. di; Aedo, A.; Loera, J. (2016). Ecopoliticas globales. Medio ambiente, bienestar y poder. Santiago de Chile: Hueders.

Girola, L. (2012). "Representaciones e imaginarios sociales, tendencias recientes en la investigación». En: Garza, E. de la; Leyva, G. (eds.) (2012). Tratado de metodología de las Ciencias Sociales: perspectivas actuales, 441-468. Universidad Autónoma Metropolitana.

Guber, R. (2011). La etnografía: método, campo y reflexividad. Buenos Aires: Siglo XXI Editores.

Gudynas, E. (2004). Ecología, economía y ética del desarrollo sostenible. 5a edición. Montevideo: Coscoroba Ediciones.

Hammersley, M.; Atkinson, P. (1994). Etnografía. Métodos de investigación. Barcelona: Paidós.

JALABERT, D. (2012). «Identidades ecosistémicas: la construcción social del paisaje lacustre en la cuenca del río Valdivia». Tesis de grado. Valdivia: Universidad Austral de Chile.

Jankilevich, S. (2003). Las cumbres mundiales sobre el ambiente. Estocolmo, Río y Johannesburgo, 30 años de Historia Ambiental. Documento de trabajo. Buenos Aires: Universidad de Belgrano.

Jiménez, V. (2012). «El estudio de caso y su implementación en la investigación». Revista Internacional de Investigación en Ciencias Sociales, 8(1), 141-150.

LefF, E. (2003). «La ecología política en América Latina: un campo en construcción». Sociedade e Estado, 18 (1), 17-40.

- (2010). «Imaginarios sociales y sustentabilidad». Cultura y Representaciones Sociales, $5(9), 42-121$.

- (2015). «Political ecology: a latin american perspective». Desenvolvimento e Meio Ambiente, 35, 29-64. <https://doi.org/10.5380/dma.v35i0.44381>.

Mata, R.; Rodríguez, I.; Cabrerizo, C.; Fernández, S. (2010). "Gestión del paisaje y gobierno del territorio. Una mirada crítica desde la región urbana de Madrid». Cuadernos de Geografía, 88, 117-140.

Ministerio del Medio Ambiente. (2014). Quinto informe nacional de biodiversidad de Chile ante el Convenio sobre la Diversidad Biológica. Santiago de Chile: MMA.

Moya, L.; VÁsquez, D. (2014). Relatos de balseros de los ríos San Pedro y Calle Calle: 1930-1960. Valdivia: Serifa.

Naredo, J. (1996). «Sobre el origen, el uso y el contenido del término sostenible». Cuadernos de Investigación Urbanistica, 41, 7-18.

O’Connor, J. (2002). «¿Es posible el capitalismo sostenible?». En: Alimonda, H. (ed.). Ecología politica: naturaleza, sociedad y utopia, 27-52. Buenos Aires: CLACSO.

Palacio, G. (2006). «Breve guía de introducción a la ecología política (Ecopol)». Gestión y Ambiente, 9 (3), 1-13.

Palma, L. (2019). Historia de la central hidroeléctrica San Pedro, el cuestionado proyecto de Colbuin SA. Valdivia: Ediciones Kultrún.

Pintos, J. (1995). «Orden social e imaginarios sociales (Una propuesta de investigación)». Papers, 45, 101-127. <https://doi.org/10.5565/rev/papers.1761>.

PNUD-GEF (2013). "Conformación de un Consejo de Desarrollo para la instalación de un modelo de gestión territorial compartida para la sostenibilidad». Programa de Naciones Unidas para el Desarrollo.

Quintana, A. (2006). "Metodología de investigación científica cualitativa». En: QuINTAna, A.; Montgomery, W. (eds.). Psicología: tópicos de actualidad. Lima: UNMSM. 
Ribeiro, G. (2011). «Antropología de la globalización. Circulación de personas, mercancías e informaciones». Cuaderno Urbano, Espacio, Cultura, Sociedad, 10 (10), 159-186.

Rocca, N. (2011). «Propuesta de plan de manejo de Conservación para el Fundo Los Bajos, dentro del Eje de Conservación Los Lagos-Riñihue». Tesis de grado. Valdivia: Universidad Austral de Chile.

Santamarina, B. (2008). "Antropología y medio ambiente. Revisión de una tradición y nuevas perspectivas de análisis en la problemática ecológica». AIBR, Revista de Antropología Iberoamericana, 3 (2), 144-184.

Santander, P. (2011). "Por qué y cómo hacer análisis de discurso». Cinta de Moebio, 41, 207-224.

SERNAGEOMIN (2003). Geología del área Los Lagos-Malalhue, Región de Los Lagos. Serie Geología Básica (81). Gobierno de Chile.

Solari, M.; Cueto, C.; Hernández, F.; Rojas, J.; Camus, P. (2011). «Procesos territoriales y bosques en la cuenca del río Valdivia (siglos XVI-XIX)». Revista de Geografia Norte Grande, 49, 45-62. <https://doi.org/10.4067/S0718-34022011000200004>.

Tecklin, D.; Catalán, R. (2005). «La gestión comunitaria de los bosques nativos en el sur de Chile: situación actual y temas en discusión». En: Catalán, R. et al. (eds.). Bosques y comunidades del sur de Chile, 19-40. Santiago de Chile: Universitaria.

Ther, F. (2012). «Antropología del territorio». Polis, 11 (32), 493-510. <https://doi.org/10.4067/S0718-65682012000200023>.

Toledo, V. (2015). «¿De qué hablamos cuando hablamos de sustentabilidad? Una propuesta ecológico-política». Interdisciplina, 3 (7), 35-55. <https://doi.org/10.22201/ceiich.24485705e.2015.7.52383>.

Tyrtania, L. (2016). «La sustentabilidad es de quien la trabaja». Cultura y Representaciones Sociales, 10 (20), 59-109.

Vergara, F. (2016). «Bosques australes y sustentabilidad: una aproximación etnográfica a los significados del bosque nativo en el valle del río San Pedro, Región de Los Ríos». Tesis de grado. Concepción: Universidad de Concepción.

Vergara-Pinto, F. y Campos, N. (2019). «Aciertos y desafíos del Paisaje de Conservación Valle Río San Pedro: Desarrollo local y conservación de la naturaleza en el sur de Chile». Revista Estudios del Desarrollo Social: Cuba y América Latina, 7 (3), 95-109.

West, P.; Igoe, J.; Brockington, D. (2006). «Parks and peoples: the social impact of protected areas». Annual Review of Anthropology, 35, 251-277. $<$ https://doi.org/10.1146/annurev.anthro.35.081705.123308>.

YIN, R. (1989). Case study research, design and metods. Londres: SAGE Publications. 